\title{
Acompanhamento de pacientes psiquiátricos na área externa hospitalar: um relato de experiência
}

\author{
Follow-up of psychiatric patients in the hospital external area: a report of experience
}

Acompañamiento de pacientes psiquiátricos en el área exterior hospitalaria: un relato de experiencia

Evelym Cristina da Silva Coelho ${ }^{1 *}$, Helloyza Halana Fernanda Aquino Pompeu ${ }^{1}$, Mário Antônio Moraes Vieira ${ }^{2}$, Alice Dayenne Moraes ${ }^{1}$, Lais Cristina Pereira da Costa Gomes ${ }^{1}$, Brenda Lorena Machado Paes ${ }^{1}$, Keila Caroline Correa Freitas ${ }^{1}$, Fernanda Cruz de Oliveira ${ }^{1}$, Claudiane Santana Silveira Amorin'1.

\section{RESUMO}

Objetivo: Relatar a experiência de uma atividade em grupo com pacientes psiquiátricos de um Setor de Internação Breve de um Hospital de Belém. Relato de Experiência: Trata-se de um estudo descritivo, do tipo relato de experiência, sobre a vivência de acadêmicos de Enfermagem na realização de atividades com pacientes psiquiátricos em regime de internação breve dentro de um hospital geral, sendo a mesma planejada e executada por uma equipe de profissionais e acadêmicos. Após a elaboração de um plano de ação, a atividade em grupo com os pacientes, foi executada em uma lanchonete próximo ao hospital. Na saúde mental, o desenvolvimento de grupos operativos permite aos pacientes o enfrentamento dos medos, das angústias e culpas e dos conflitos presentes no cotidiano. Desta forma, é uma estratégia eficaz possível de auxiliar no tratamento destes pacientes, além de ser de baixo custo. Nesta experiência, foi possível a observação de fenômenos durante a execução como o processo de busca de autonomia e valorização da cidadania, perdidas com o processo de crise. Considerações finais: Por meio das boas práticas no cuidar dos pacientes deste estudo, foi possível observar a necessidade de se realizar atividades que coloquem os pacientes como um sujeito protagonista inserido na sociedade em que vive, promovendo um cuidar pautado na humanização.

Palavra-chave: Saúde Mental, Promoção da Saúde, Enfermagem Psiquiátrica.

\begin{abstract}
Objective: to report an experience of a group exercise with psychiatric patients of a Hospital of Brief Hospital of Belém. Experience report: This is a descriptive study, of the type of experience, about the experience of nursing academics in conducting psychiatric activities in a short-stay hospital in a general hospital, and it is planned and executed by a team of professionals and academics. After elaborating a plan of action, a group walk with patients was launched at a snack bar in the hospital. In mental health, the development of surgical groups allows patients to face the fears, anxieties and guilts and conflicts present in daily life. In this way, it is an effective strategy of help without the treatment of the patients, besides being low cost. In this experience, it was possible to verify the process during the execution as a process of seeking autonomy and valorization of citizenship, lost through the crisis process. Final considerations: Through the good practices in the care of the patients of this study, it was possible to observe the accomplishment of activities that put the patients as a protagonist in the society that lives, promoting a care in the humanization.
\end{abstract}

Keywords: Mental health, Health Promotion, Psychiatric Nursing.

1 Universidade do Estado do Pará (UEPA). * E-mail: evelym titina@hotmail.com

2 Universidade Castelo Branco do Rio de Janeiro-RJ. 


\section{RESUMEN}

Objetivo: relatar una experiencia de un ejercicio en grupo con pacientes psiquiátricos de un Sector de Internación Breve de un Hospital de Belém. Relato del experiencia: Se trata de un estudio descriptivo, del tipo de experiencia, sobre la vivencia de académicos de enfermería en la realización de actividades psiquiátricas en régimen de internación breve dentro de un hospital general, siendo la misma planeada y ejecutada por un equipo de profesionales y académicos. Después de la elaboración de un plan de acción, un paseo en grupo con pacientes, fue lanzado en una cafetería en el hospital. En la salud mental, el desarrollo de grupos quirúrgicos permite a los pacientes el enfrentamiento de los miedos, de las angustias y culpas y de los conflictos presentes en el cotidiano. De esta forma, es una estrategia eficaz de ayuda sin el tratamiento de los pacientes, además de ser de bajo costo. En esta experiencia, fue posible una verificación de proceso durante la ejecución como proceso de búsqueda de autonomía y valorización de la ciudadanía, perdidas con el proceso de crisis. Consideraciones finales: Por medio de las buenas prácticas en el cuidado de los pacientes de este estudio, fue posible observar la realización de actividades que coloquen a los pacientes como un sujeto protagonista en la sociedad que vive, promoviendo un cuidado en la humanización.

Palabra clave: Salud mental, Promoción de la Salud, Enfermería Psiquiátrica.

\section{INTRODUÇÃO}

Tradicionalmente, a assistência à saúde mental esteve pautada no isolamento, na tutela, na vigilância, na repressão e na disciplina e esteve baseada no modelo hospitalocêntrico de assistência. O manicômio era o espaço onde se desenvolviam essas ações, o único reservado para a pessoa que não possuía uma razão comum e não se comportava como um indivíduo dito "normal", assim, os pacientes psiquiátricos se transformavam em pessoas sem direitos (MELO, 2012).

Nessa perspectiva, o movimento da Reforma Psiquiátrica trouxe consigo uma verdadeira revolução teórico-conceitual, surgindo como resposta aos modos de como as pessoas com sofrimento psíquico eram tratadas nos hospitais psiquiátricos, em favor da mudança dos modelos de atenção e gestão das práticas de saúde, defesa da saúde coletiva, equidade na oferta dos serviços e protagonismo dos trabalhadores e usuários dos serviços de saúde (BRASIL, 2013).

A partir dessa renovação do modelo assistencial em saúde mental, boas práticas em saúde mental foram sendo estruturadas. Uma dessas práticas é o grupo terapêutico, muito utilizado nos Centros de Apoio Psicossociais (CAPS), trata-se de uma ferramenta que pode ser desenvolvida com pacientes internados, principalmente, por proporcionar espaços onde seja possível fugir da enclausuração que simboliza estar hospitalizado (HERNANDES et al., 2011).

Em estudo realizado na cidade de Minas Gerais, mostrou que 56,6\% dos usuários do CAPS pertenciam a pacientes do sexo masculino, 474 pacientes, sendo esse número em torno de $81,3 \%$, tinham idade entre 18 e 50 anos, Em relação ao histórico psiquiátrico, 18,3\% foram atendidos pela primeira vez em serviços de saúde mental e $64,7 \%$ tinham passado de alguma modalidade de tratamento psiquiátrico ou de pelo menos uma internação hospitalar ( MANGUALDE et al., 2013).

Para Pichon-Riviére (2005), o grupo é caracterizado pela reunião de pessoas no mesmo tempo e espaço para a efetivação de uma tarefa, a partir de atribuição de papeis. Desta forma, os grupos terapêuticos não tratam apenas de mantê-los ocupados, mas de mostrar que mesmo se encontrando dentro de uma clínica, é possível ter uma identidade.

Spadini e Souza (2006) afirmam que os grupos são ferramentas terapêuticas que possibilitam a recriação de ambientes familiares, bem como sociais, o que permite o aperfeiçoamento das habilidades dos participantes. Logo, a promoção de atividades grupais é uma opção terapêutica com múltiplas finalidades, como: incentivar a realização de atividades físicas, inserir pacientes em ambiente mais propício à socialização, promover contato com o ambiente externo/ natureza se houver possibilidade e reavivar sua autonomia, pois é ofertada a ele a opção de participar das atividades propostas ou não (RIBEIRO et al., 2014). 
Além disso, apesar da prática de atividade em grupo ser centrada nos pacientes, os profissionais que as coordenam são atores essenciais, pois o sucesso depende dos princípios teóricos e práticos destes profissionais (Ribeiro et al., 2014). Portanto, estudos a respeito das práticas de atividades em grupos na área da saúde mental, tornam-se essenciais para auxiliar a prática profissional.

Nesse sentido, considerando a importância dessas atividades para o tratamento não farmacológico de pacientes com transtornos mentais, este estudo propõe-se a relatar o desenvolvimento de uma atividade em grupo, a fim de contribuir para o aprimoramento da assistência prestada aos pacientes com transtornos mentais.

\section{RELATO DE EXPERIÊNCIA}

Trata-se de um estudo descritivo, do tipo relato de experiência, sobre a vivência de acadêmicas de Enfermagem na realização de uma atividade em grupo com pacientes psiquiátricos. Este relato foi realizado durante as aulas práticas do componente curricular Saúde Mental II no Setor de Internação Breve (SIB) de um Hospital Geral Público do município de Belém-PA que, além do SIB, possui uma porta de entrada para urgências e emergências de pessoas com sofrimento ou transtorno mental.

O SIB permite a permanência de pacientes com sintomas agudos, por um tempo de aproximadamente 30 dias, o qual possui 15 leitos masculinos e 15 femininos, além da atuação de uma equipe multiprofissional, sendo eles: médicos psiquiatras, psicólogos, enfermeiros, educador físico, terapeuta ocupacional, assistente social e técnicos de enfermagem.

Desta forma, a atividade em grupo foi realizada com pacientes do SIB do referido hospital, sendo a mesma planejada e executada por uma equipe de profissionais e acadêmicos. Essa vivência será descrita desde os passos de planejamento até a execução da mesma:

\section{1 - Planejamento da tarefa}

Primeiramente houve uma reunião entre os acadêmicos e seu preceptor com a equipe multiprofissional, para apresentar, discutir e avaliar a proposta de uma atividade grupal na área externa do hospital. Foi solicitado parecer técnico a respeito das vantagens para o paciente que está em regime de atendimento breve internado na clínica.

Após esta reunião, aconteceu a seleção dos critérios de inclusão para participar da atividade, considerando o perfil do grupo terapêutico e os riscos da atividade a ser realizada, sendo os critérios: pacientes em remissão dos sintomas agudos, hipoativos, com isolamento social importante, sem potencial para agressividade, sem risco de fuga, não ser dependente químico, sem restrição alimentar, os que verbalizavam o desejo de participar e os que obedeciam aos comandos espontaneamente.

Foram também discutidos os principais cuidados que deveriam ser adotados pela equipe durante o trajeto como: caminhar pelos lados, chamar o paciente sempre pelo seu nome ou como gostaria de ser chamado, a fim de garantir sua individualização. Frente as possíveis não conformidades relacionadas ao comportamento agressivo e dificuldade de entendimento, houve a necessidade de um planejamento de intervenção mais contenedora que transcendesse o manejo planejado e neste caso, se utilizaria a contenção mecânica. Também foi previsto neste translado a composição de um kit de materiais com medicamentos injetáveis, previamente prescritos, esfigmomanômetro e estetoscópio e tiras de contenção para atendimento de emergências.

\section{2 - Execução da atividade externa}

No dia da atividade, aconteceu primeiramente uma conversa entre a equipe e a seleção prévia dos pacientes e, após isso, foi perguntado aos pacientes se os mesmos gostariam de fazer parte do grupo. Após 
esta seleção, foi compartilhado com eles as regras que deveriam cumprir contratualizando que deveriam buscar atender os comandos da equipe responsável pelo seu tratamento. Além disso, foi garantida a retirada da roupa de internação, trocando-as por vestimentas habituais cedidas pela equipe, de maneira que não 0 discrimine como pacientes psiquiátricos fora do local de tratamento.

Notou-se o entusiasmo dos pacientes quando foram direcionados para trocarem de roupa, este fato denota como é importante preservar a dignidade, integridade e autonomia do paciente que, mesmo em internação hospitalar, tem como necessidade humana básica manter uma autoimagem e autoestima adequadas. Hernandes et al.,(2011) afirmam que ações como essas visam mudanças no tratamento do sofrimento psíquico, sobretudo, pela valorização da cidadania e da cultura da participação, culminando no exercício da democracia.

O local de escolha para a atividade foi uma lanchonete próxima ao hospital e lá foi atendido o desejo do paciente quanto sua escolha do lanche, garantindo seu desejo dentro de um limite mínimo financeiro do grupo organizador, mostrando a ele a importância da autonomia para garantir o seu próprio sustento e manutenção das suas vontades e desejos. Além disso, enquanto vivenciavam aquela experiência de socialização, foi demonstrada a importância do relacionamento interpessoal com a comunidade que estava ali, exercitando o princípio sine qua non da cidadania.

Na realização do lanche percebeu-se que os pacientes interagiam, conversavam sobre gostos alimentares, aceitaram registrar o momento com fotos e foram bastantes adeptos a atividade, até mesmo um dos pacientes, que se mostrava introspectivo respondeu, ainda que pouco, quando lhe era instigado a falar. Além disso, o companheirismo e sentimento de solidariedade ao partilhar o lanche, por exemplo, foi perceptível entre os próprios pacientes.

No retorno, antes de voltar ao SIB, foi realizada uma avaliação com os pacientes, com o objetivo de exteriorizar os valores internalizados da tarefa realizada de forma objetiva, podendo ser por expressão verbal, gestual, musical ou mesmo sem nenhuma manifestação de forma que participe do processo de avaliação, sendo este momento a catarse do grupo terapêutico.

Assim, os mesmos puderam falar sobre a atividade e dentre os relatos, destacou-se o fato deles terem gostado da roupa, das diferenças entre o lanche do hospital e o local, além da atenção que foi dada a eles. Essa é uma forma relevante de avaliar a eficácia da atividade sobre os pacientes uma vez que é pautada no relato do próprio beneficiado pela atividade e ainda é muito importante dar voz ao paciente, estimulando-o a exteriorizar seus pensamentos.

Após avaliação da atividade, os pacientes foram conduzidos novamente à enfermaria e, antes de adentrarem o local, foram até um espaço restrito para a retirada da roupa comum e colocação do uniforme exigido pela instituição. Desta forma, os pacientes foram orientados para que seguissem seu tratamento e que atividades como a proposta permitem auxiliar sua terapêutica, bem como avaliar seu estado e Ihe proporcionar mais autonomia, autocontrole e inserção social a fim de que este possa receber sua alta melhorada e continuar seu tratamento nos Centros de Atenção Psicossocial.

\section{CONSIDERAÇÕES FINAIS}

Por meio das boas práticas no cuidar dos pacientes deste estudo, foi possível observar a real necessidade de se realizarem atividades as quais coloquem os pacientes como um sujeito totalmente inserido na sociedade em que ele vive, promovendo um cuidar pautado na humanização.

Dessa forma, as atividades grupais fizeram os pacientes socializarem entre si e com a equipe que estava os acompanhando de forma holística e horizontal, onde todos eram iguais, cidadãos pertencentes à uma sociedade, estimulando a fala e a interação social. Assim, as práticas de atividades em grupos operativos trazem de volta ao paciente com transtorno mental um direito que lhes deve ser sempre garantido, o direito à cidadania. 
Diante desse contexto, com esse trabalho foi possível garantir uma real integração entre ensino-serviçopaciente e assim, sugere-se a promoção demais atividades como essas dentro dos hospitais, a fim de suscitar ao paciente com transtorno mental que está internado o interesse pela comunicação, pela interação social, pelo seu ser social e por meio da aproximação entre as partes envolvidas, motivar uma avaliação visando concretizar a garantia da efetividade dos princípios básicos do Sistema Único de Saúde proporcionando especialmente uma assistência integral ao paciente.

\section{REFERENCIAS}

1. BRASIL. Caderno de Atenção Básica: Saúde mental. Brasília: Ministério da Saúde, 2013.

2. HERNANDES KM, BRUNIERA MS, LUZIO CA et al. Oficina na Atenção Psicossocial: Experimentações com a palavra. Revista de Psicologia da UNESP. 2011; 10(1): 89-99.

3. MELO AMC. Apontamentos sobre a reforma psiquiátrica no brasil. Cadernos Brasileiros de Saúde Mental. Florianópolis. 2012; 8(9): 84-95.

4. MANGUALDE AAS, BOTELHO CC, SOARES MR et al. Perfil epidemiológico dos pacientes atendidos em um Centro de Atenção Psicossocial. Mental Revista de Saúde Mental e Subjetividade da UNIPAC. 2012; 10(19) :235-248.

5. PICHON-RIVIÉRE E. O processo grupal. Matins e Fontes. 2005.

6. RIBEIRO LA, MARIN LL, SILVA MTD et al. Atividades Grupais em Saúde Mental. Revista Baiana de Enfermagem. 2014; 28 (3).

7. SPADINI LS, SOUZA MCBM. Grupos Realizados por Enfermeiros na Área de Saúde Mental. Escola Anna Nery Revista de Enfermagem. 2006; 10 (1): 132-138. 